\title{
A low-power current bleeding mixer with improved LO-RF isolation for ZigBee application
}

\begin{abstract}
This paper present a low power current bleeding CMOS mixer with high LO-RF isolation for ZigBee application. The proposed mixer uses current reuse technique with self-biased transconductance stage to increase the conversion gain while substantially reducing the DC power dissipation. A NMOS current bleeding transistor and load resistor is integrated between the RF transconductance and LO switching stage to improve the LO-RF isolation. This mixer is verified in $0.13 \mathrm{\varepsilon m}$ standard CMOS technology. The simulation result shows a high conversion gain (CG) of $12 \mathrm{~dB}, 1 \mathrm{~dB}$ compression point (P1dB) of $-13.4 \mathrm{dBm}$, thirdorder intercept point (IIP3) of $-4.3 \mathrm{dBm}$ and a noise figure (NF) of $15.45 \mathrm{~dB}$. The circuit consumes 664 EA current from $1.2 \mathrm{~V}$ power supply and LO-RF isolation is improved by 25 $\mathrm{dB}$.
\end{abstract}

Keyword: Mixer; Current-reused; LO-RF isolation; CMOS; Conversion gain; ZigBee 